\title{
Hyperglycemia During Pregnancy Depends on Numerous Factors (Hypertension During Pregnancy, Previous Birth of a Fetus over 4 kg, Hirsutism - Bulgarian Screening, 2019)
}

\author{
Anna-Maria Borissova ${ }^{1,2}$, Boyana Trifonova ${ }^{1,2}$, Lilia Dakovska ${ }^{1}$, Eugenia Michaylova ${ }^{3}$, \\ Mircho Vukov ${ }^{1}$ \\ ${ }^{1}$ Clinic of Endocrinology, University Hospital Sofiamed, Sofia, Bulgaria \\ ${ }^{2}$ Faculty of Medicine, Sofia University Saint Kliment Ohridski, Sofia, Bulgaria \\ ${ }^{3}$ Medical Laboratory Bodimed, Sofia, Bulgaria
}

Email address:

boianatri@abv.bg (B. Trifonova)

\section{To cite this article:}

Anna-Maria Borissova, Boyana Trifonova, Lilia Dakovska, Eugenia Michaylova, Mircho Vukov. Hyperglycemia During Pregnancy Depends on Numerous Factors (Hypertension During Pregnancy, Previous Birth of a Fetus over 4 kg, Hirsutism - Bulgarian Screening, 2019). International Journal of Diabetes and Endocrinology. Vol. 6, No. 2, 2021, pp. 69-75. doi: 10.11648/j.ijde.20210602.13

Received: April 3, 2021; Accepted: April 19, 2021; Published: April 29, 2021

\begin{abstract}
Gestational diabetes mellitus (GDM) starts during pregnancy and can increase the risk of adverse pregnancy outcomes, as well as be the cause of a number of diseases in the years after birth in both the mother and the fetus. The aim is to study in the Bulgarian population of pregnant women the relationship between hyperglycemia during pregnancy and some factors - arterial hypertension (AH), previous macrosomic baby weighing $4.5 \mathrm{~kg}$ or above, hirsutism. Material: A cross-sectional multicenter population-based study which included 547 pregnant women, with average age 30.49 $\pm 5,12$ years was conducted in 84 settlements in Bulgaria. Methods: A questionnaire was completed, blood pressure was measured (Guideline of ESC/ESH, 2018), BMI was calculated. A two-hour, $75 \mathrm{~g}$ oral glucose tolerance test (OGTT) was performed. Glucose was quantitatively determined using enzymatic reference method with hexokinase (Roche reagent) on Cobas e501 analyzer. The statistical analysis was performed using standard SPSS 13.0 for Windows. Results: The incidence of Hyperglycemia for the whole group of pregnant women was $14.4 \%$ (79/547), up to 24 gestational week (g.w.) it was 5.3\% (29/547) and after 24 g.w. - 9.1\% (50/547), P $<0.01$. Of all screened pregnant women, 2.4\% (13/547) developed AH during the current pregnancy. Hyperglycemia was found in $38.5 \%(5 / 13)$ of the women with $\mathrm{AH}$ and in $13.9 \%$ (74/534) of the women without $\mathrm{AH}, \mathrm{P}<0.028$. In $3.29 \%$ of the pregnant women (18/547) a macrosomic baby weighing $4.5 \mathrm{~kg}$ or above was found in a previous pregnancy. Hyperglycemia was present in $38.9 \%$ (7/18) of women who gave birth to a large fetus against $13.6 \%$ (72/529) of women who gave birth to a fetus under $4 \mathrm{~kg}, \mathrm{P}<0.008$. Hirsutism was reported in $7.9 \%$ (43/547) of the studied pregnant women. Hirsutism is twice as common in pregnant women with Hyperglycemia - 13.9\% (11/79) versus hirsutism in those with Normoglycemia $-6.8 \%(32 / 468), \mathrm{P}<0.049$. There was a significant correlation between Hyperglycemia and gestational age $(\mathrm{P}<0.006)$, previous fetal birth over $4 \mathrm{~kg}(\mathrm{P}<0.03)$, AH during the current pregnancy $(\mathrm{P}<$ $0.01)$, presence of hirsutism $(\mathrm{P}<0.03)$. Conclusion: A good knowledge of all risk factors associated with the development of glucose intolerance and GDM could play an important role in the early diagnosis of this common disorder during pregnancy.
\end{abstract}

Keywords: Pregnancy, Hyperglycemia, Arterial Hypertension, Macrosomic Baby Weighing $4.5 \mathrm{~kg}$ or Above, Hirsutism

\section{Introduction}

Hyperglycemia is one of the most common conditions during pregnancy [1]. According to classification of the International Federation of Gynecology and Obstetrics FIGO [2] and the World Health Organization - WHO [3], hyperglycemia detected for the first time during pregnancy may be related to: 1) Diabetes in Pregnancy (DIP) - Diabetes diagnosed for the first time during pregnancy according to generally accepted population diagnostic criteria for diabetes (standard oGTT $75 \mathrm{~g}$ glucose): fasting plasma glucose $\geq 7.0$ $\mathrm{mmol} / \mathrm{L}$ or 2 nd hour plasma glucose $\geq 11.1 \mathrm{mmol} / \mathrm{L}$ or $\mathrm{HbAlc} \geq 48 \mathrm{mmol} / \mathrm{mol}$ or $\geq 6.5 \%$ and 2) Gestational diabetes (GDM) - hyperglycemia above the diagnostic threshold for 
gestational diabetes (standard oGTT $75 \mathrm{~g}$ glucose): fasting plasma glucose $5.1-6.9 \mathrm{mmol} / \mathrm{L}, 1^{\text {st }}$ hour plasma glucose $\geq$ $10.0 \mathrm{mmol} / \mathrm{L}, 2^{\text {nd }}$ hour plasma glucose $8.5-11.0 \mathrm{mmol} / \mathrm{L}[2$, 3]. As early as 2015, the National Institute for Health and Care Excellence-NICE [4] published criteria for diagnosing GDM: plasma fasting blood glucose $(\mathrm{FBG}) \geq 5.6 \mathrm{mmol} / \mathrm{L}$ or plasma blood glucose at 120 minutes $\geq 7.8 \mathrm{mmol} / \mathrm{L}$. The NICE Recommendations also include instructions for establishing of pregnancy up to 10-16 gestational week (g.w.) to perform oral glucose tolerance test (oGTT) for detection of undiagnosed diabetes. Additionally, in case of normal results, a second oGTT should be performed at the first screening at 24-28 g.w. to open the GDM [4]. GDM started during pregnancy, and it is clear that it did not exist before pregnancy [5].

About half of the women with GDM develop type 2 diabetes mellitus (T2D) 5-10 years after giving birth. It is important for women with Diabetes in pregnancy (DIP) or with GDM to monitor their blood glucose (BG) levels regularly in order to reduce the risk of adverse pregnancy outcomes [1].

Children of mothers with diabetes, especially if they also have CVD or diabetic complications, are at high risk of developing CVD in childhood or adolescence [6]. GDM also increases the risk of diabetes and obesity in the offspring during their childhood and adolescence [7] and for mothers, the risk of T2D increases significantly [8]. Recent publications have shown, that there is a link between failed pregnancies and T2D, and this link becomes stronger as the number of failed pregnancies increases [9]. GDM increases the risk of adverse pregnancy outcomes, such as pre-eclampsia, more frequent maternal cesarean section, neonatal hypoglycemia, large fetus, birth trauma for the fetus [10]. It is believed that GDM increases the risk of developing T2D in the offspring through intrauterine mechanisms [11]. In diabetics, the intrauterine environment can lead to placental dysfunction and hormonal disorders $[12,13]$. Various genetic and epigenetic factors, as well as environmental factors through various complex mechanisms contribute to the development of GDM. Physiologically the metabolic activity during pregnancy is high so the maintenance of glucose homeostasis is of great importance. The $\beta$-cells of the pancreas fail to compensate for the necessary energy needs, significant insulin resistance (IR) develops and too much glucose goes to the growing fetus. Mild chronic inflammation, impaired gluconeogenesis, oxidative stress, and some placental factors also contribute to the development of GDM. All this requires the introduction of timely and successful strategies for prevention of GDM [14].

That is why the timely diagnosis of this common complication of pregnancy is extremely important. The international recommendations specifically indicate the need for screening of pregnant women in the presence of risk factors for GDM, among which the most frequently mentioned are obesity, previous GDM, family burden with diabetes - first degree relatives $[2,3,4]$. In addition to these major risk factors for the development of hyperglycemia during pregnancy, there are other no less important that influence the manifestation of this frequent complication during pregnancy.
The aim of the present study was to investigate the relationship between Hyperglycemia during pregnancy and the sequence of pregnancy, arterial hypertension, large fetus over $4 \mathrm{~kg}$ at birth, hirsutism, reproductive problems or adverse outcome of previous pregnancies among the Bulgarian population of pregnant women.

\section{Material}

The mean age of the studied 547 pregnant women was $30.49 \pm 5,12$ years, Median 30.00 (minimum 18 - maximun 47). BMI before pregnancy was $23.36 \pm 5.05 \mathrm{~kg} / \mathrm{m}^{2}$, Median 22.06, and BMI at the time of screening was $25.91 \pm 5.11 \mathrm{~kg} / \mathrm{m}^{2}$, Median 25.25. Distribution of the studied 547 pregnant women by trimesters and gestational week (g.w.) is as follows: first - $110(20.1 \%)$ - up to 12 g.w, second - $276(50.5 \%)$ 13-24 g.w, third - 161 (29.4\%) - after 24 g.w.

According to the sequence of pregnancies, women were divided into six categories, Table 1.

Table 1. Sequence of pregnancies among the surveyed 547 pregnant women.

\begin{tabular}{lllllll}
\hline Sequence of Pregnancy & first & second & third & four & five & six \\
\hline Number & 245 & 216 & 57 & 19 & 9 & 1 \\
Percentage & 44.8 & 39.5 & 10.4 & 3.5 & 1.6 & 0.2 \\
\hline
\end{tabular}

As can be seen, $84.3 \%$ of the pregnant women had first and second pregnancy, and the remaining $15.7 \%$ were with sequence pregnancy. Two thirds of them had third consecutive pregnancy $(10.4 \%)$ and only one third - fourth to sixth $(5.3 \%)$.

All participants signed informed consent, confirmed by the local Ethics Commission at Sofiamed University Hospital, Sofia University "Saint Kliment Ohridski". It was prepared in accordance with the ethical standards according to the Helsinki Declaration-1964 and later additions [15].

Each pregnant woman filled in a questionnaire with the assistance of a specially designated medical person from the "face to face" team in order to correctly collect data on pregnancy history, past healthy history, family history for diabetes, hypertension, thyroid and chronic kidney disease, current and past medication, smoking. All participants were Caucasian, with no evidence of liver or kidney disease or evidence of malabsorption.

\section{Methods}

\subsection{The Weight and Height Resp. BMI-Before and During Pregnancy; Arterial Pressure}

After completing the personal Questionnaire, the current weight and height of each pregnant woman were measured and filled in the Questionnaire together with the weight before pregnancy. The body mass index (BMI $-\mathrm{kg} / \mathrm{m}^{2}$ ) before pregnancy and the current one at the time of the study were calculated. Mean weight before pregnancy was $63.68 \pm 14.36 \mathrm{~kg}$, Median 60.00 (minimum 40 - maximun 166), and weight at the time of screening was $70.59 \pm 14.50 \mathrm{~kg}$, Median 68.00 (minimum 41 - maximun 166). The body mass index (BMI) before pregnancy was $23.36 \pm 5.05 \mathrm{~kg} / \mathrm{m}^{2}$, Median 22.06 (minimum 
15.24 - maximun 63.25), and BMI at the time of screening was $25.91 \pm 5.11 \mathrm{~kg} / \mathrm{m}^{2}$, Median 25.25 (minimum 16.51 - maximun 63.25). At addition Arterial blood pressure was measured in the sitting position after a 5-minute rest according with the last Guideline of European Society of Cardiology (ESC) and the European Society of Hypertension (ESH) [16].

\subsection{Laboratory Analysis}

A two-hour, $75 \mathrm{~g}$ oral glucose tolerance test (OGTT) was performed. Venous blood was drawn at 0,60 and 120 minutes in plasma tubes, containing Na2EDTA and $\mathrm{NaF}$, as an inhibitor of glycolysis for stability of glucose in the samples. The samples were transported to the laboratory after centrifugation. All of the samples were analysed in one Central laboratory on the day of the blood sampling. Glucose was quantitatively determined using enzymatic reference method with hexokinase (Roche reagent) on Cobas e501 analyzer. The results were in $\mathrm{mmol} / \mathrm{l}$. Established precision using human samples and controls:

1) Intra assay: Level $1(n=6) C V=1.12 \%$; Level $2(n=6)$ $\mathrm{CV}=0.42 \%$

2) Inter assay: Level $1(\mathrm{n}=30) \mathrm{CV}=1.25 \%$; Level $2(\mathrm{n}=30)$ $\mathrm{CV}=1.58 \%$

3) Two levels Intralaboratory quality control on a daily basis was performed.

The Laboratory participates in two EQA systems Bulgarian EQAS and INSTAND and has certificates for this parameter.

\subsection{The Statistical Analysis}

The statistical analysis was performed using standard SPSS 13.0 for Windows: descriptive statistics (mean, medians, standard deviation), correlation analysis and analysis of variance (ANOVA, post-hoc test - with Bonferroni alpha correction), using parametrical and non-parametrical methods, including - Chi-Square Test, Fisher's Exact Test, Kolmogorov-Smirnov, Shapiro-Wilk Tests, Levene's Test for Equality of Variances, Student's t-test, Kruskal-Wallis test and Mann -Whitney test. All quantitative variables were presented as mean with standard deviation, median or percentage (unless specified otherwise), p values below 0.05 were accepted as statistically significant.

\section{Results}

\subsection{Sequence of Pregnancy}

The incidence of Hyperglycemia for the whole group of pregnant women was $14.4 \%(79 / 547)$, and up to 24 g.w. was $5.3 \%(29 / 547)$ and after 24 g.w. $-9.1 \%$ (50/547), $\mathrm{P}<0.01$. It is noteworthy that with each successive pregnancy the percentage of women with Hyperglycemia increases (from $14.3 \%$ in the first to $36.8 \%$ in the fourth pregnancy), NS. For the period up to $24 \mathrm{~g}$.w. no significant dynamics is observed in this frequency, but after 24 g.w. the incidence of Hyperglycemia increased from $9.4 \%$ in the first pregnancy to $31.6 \%$ in the fourth (NS), Table 2.

Table 2. Sequence of pregnancy and frequency of Hyperglycemia in total, up to 24 g.w. and after 24 g.w. periods

\begin{tabular}{llll}
\hline $\begin{array}{l}\text { Sequence of } \\
\text { Pregnancy }\end{array}$ & $\begin{array}{l}\text { Frequency of Hyperglycemia in the } \\
\text { whole group (number, percentage) }\end{array}$ & $\begin{array}{l}\text { Frequency of hyperglycemia up to 24 g.w. } \\
\text { (number, percentage) }\end{array}$ & $\begin{array}{l}\text { Frequency of hyperglycemia after 24 g.w. } \\
\text { (number, percentage) }\end{array}$ \\
\hline First & $35(14.3 \%)$ & $12(4.9 \%)$ & $23(9.4 \%)$ \\
Second & $25(11.6 \%)$ & $13(6 \%)$ & $12(5.6 \%)$ \\
Third & $9(15.8 \%)$ & $2(3.5 \%)$ & $7(12.3 \%)$ \\
Fourth & $7(36.6 \%)$ & $1(5.0 \%)$ & $6(31.6 \%)$ \\
Fifth & $2(22.2 \%)$ & $1(11.1 \%)$ & $1(11.1 \%)$ \\
Sixth & 1 & & 1 \\
Total & $79(14.4 \%)$ & $29(5.3 \%)$ & $50(9.1 \%)$ \\
\hline
\end{tabular}

\subsection{Arterial Hypertension Before Pregnancy}

Previous arterial hypertension $(\mathrm{AH})$ before the current pregnancy was found in $4 \%(22 / 547)$ of all screened pregnant women. In this group of women with previous $\mathrm{AH}, 18.2 \%$ (4/22) had Hyperglycemia versus $14.3 \%$ (75/525) in women without AH before the current pregnancy, NS. Hyperglycemia up to 24 g.w. was found in $9.1 \%(2 / 22)$ of women with previous AH versus $5.1 \%(27 / 525)$ of those without previous AH, NS. Hyperglycemia after 24 g.w. proved in 9.1\% (2/22) of women with previous AH versus $8.8 \%(48 / 525)$ in those without previous $\mathrm{AH}$, NS. It can be summarized that pre-pregnancy $\mathrm{AH}$ is not a significant factor in the occurrence of disturbances in glucose tolerance during pregnancy.

\subsection{Arterial Hypertension During Pregnancy}

Of all pregnant women screened, 2.4\% (13/547) developed $\mathrm{AH}$ during the current pregnancy. Hyperglycemia was found in $38.5 \%(5 / 13)$ of women with AH versus $13.9 \%$ (74/534) of women without $\mathrm{AH}, \mathrm{P}<0.028$. Hyperglycemia up to 24 g.w. was found in $7.7 \%(1 / 13)$ of pregnant women with $\mathrm{AH}$ versus $5.2 \%(28 / 534)$ of pregnant women without AH, NS. Hyperglycemia after 24 g.w. was found in 30.8\% (4/13) of pregnant women with $\mathrm{AH}$ versus $8.6 \%$ (46/534) of pregnant women without $\mathrm{AH}, \mathrm{P}<0.024$.

\subsection{Previous Macrosomic Baby Weighing $4.5 \mathrm{~kg}$ or Above}

In $3.29 \%$ of the pregnant women (18/547) a fetus over $4 \mathrm{~kg}$ was found in a previous pregnancy. Hyperglycemia was present in $38.9 \%(7 / 18)$ of the women who gave birth to a large fetus over $4 \mathrm{~kg}$ versus $13.6 \%(72 / 529)$ of the women who gave birth to a fetus less than $4 \mathrm{~kg}$ in a previous pregnancy, $\mathrm{P}<$ 0.008 . Hyperglycemia up to $24 \mathrm{~g}$.w. was found in $22.2 \%(4 / 18)$ of the women who gave birth to a fetus over $4 \mathrm{~kg}$ against $4.7 \%$ $(25 / 529)$ of the women who gave birth to a fetus under $4 \mathrm{~kg}$ in a previous pregnancy, $\mathrm{P}<0.012$. Hyperglycemia after 24 g.w. 
was registered in $16.7 \%(3 / 18)$ of the women who gave birth to a fetus over $4 \mathrm{~kg}$ in a previous pregnancy against $8.6 \%$ $(47 / 547)$ of the women who gave birth to a fetus under $4 \mathrm{~kg}$, NS.

\subsection{Hirsutism}

Hirsutism was found in $7.9 \%(43 / 547)$ of the screened pregnant women, while in $92.1 \%(504 / 547)$ of them there wasn't any. It turned out that hirsutism was twice as common in pregnant women with Hyperglycemia - 13.9\% (11/79) against hirsutism in pregnant women with Normoglycemia $6.8 \%$ (32/468), $\mathrm{P}<0.049$. In pregnant women up to 24 g.w. Hyperglycemia is twice as common in the presence of hirsutism $17.2 \%(5 / 29)$ versus $7.3 \%(38 / 518)$ in its absence, NS. Pregnant women after 24 g.w. have Hyperglycemia $62 \%$ more often in the presence of hirsutism - $12 \%(6 / 50)$ compared to pregnant women without hirsutism - 7.4\% (37/497), NS (Table 3).

Table 3. Distribution of cases of Hyperglycemia during pregnancy in total, before and after 24 g.w. among women with and without hirsutism

\begin{tabular}{lll}
\hline Hirsutism & Yes & No \\
\hline 468 pregnants with Normoglycemia Number (\%) & $32 / 468(6.8)^{*}$ & $436 / 468(93.2)$ \\
79 pregnants with Hyperglycemia Number (\%) & $11 / 79(13.9)^{*}$ & $68 / 79(86,1 \%)$ \\
518 pregnants with Normoglycemia up to 24 g.w. Number (\%) & $38 / 518(7.3)$ & $480 / 518(92.7 \%)$ \\
29 pregnants with Hyperglycemia up to 24 g.w. Number (\%) & $5 / 29(17.2)$ & $24 / 29(82.8)$ \\
497 pregnants with Normoglycemia after 24 g.w. Number (\%) & $37 / 497(7.4)$ & $460 / 497(92.6)$ \\
50 pregnants with Hyperglycemia after 24 g.w. Number (\%) & $6 / 50(12.0)$ & $45 / 50(88.0)$ \\
\hline
\end{tabular}

\subsection{Polycystic Ovary Syndrome}

Anamnestic data for previous polycystic ovary syndrome (PCOS) were obtained in $11.2 \%(61 / 547)$ of the studied pregnant women. It turned out that in these 61 pregnant women with previous PCOS, Hyperglycemia was present in $14.8 \%(9 / 61)$ - in the subgroup up to 24 g.w. - in $11.5 \%(7 / 61)$ and after 24 g.w. - in 3.3\% (2/61). Table 4 presents the distribution of pregnant women with and without previous PCOS in the groups with Hyperglycemia resp. Normoglycemia - total, before and after 24 g.w. No significant difference was found between the groups mainly due to the very small number of pregnant women with Hyperglycemia on the background of previous PCOS - only 9.

Table 4. Incidence of Hyperglycemia in pregnant women with and without previous PCOS - total, before and after 24 g.w.

\begin{tabular}{lll}
\hline Polycystic ovary syndrome & Yes (61/547, 11.2\%) & No (486/547, 88.8\%) \\
\hline 468 pregnants with Normoglycemia Number (\%) & $52 / 468(11.1)$ & $416 / 468(88.9)$ \\
79 pregnants with Hyperglycemia Number (\%) & $9 / 79(11.4)$ & $70 / 79(88,6)$ \\
518 pregnants with Normoglycemia up to 24 g.w. Number (\%) & $54 / 518(10.4)$ & $464 / 518(89.6)$ \\
29 pregnants with Hyperglycemia up to 24 g.w. Number (\%) & $7 / 29(24.1)$ & $22 / 29(75.9)$ \\
497 pregnants with Normoglycemia after 24 g.w. Number (\%) & $59 / 497(11.8)$ & $438 / 497(88.2)$ \\
50 pregnants with Hyperglycemia after 24 g.w. Number (\%) & $2 / 50(4.0)$ & $48 / 50(96.0)$ \\
\hline
\end{tabular}

\subsection{Reproductive Problems}

Reproductive problems were found in $21.8 \%(119 / 547)$ of pregnant women. Hyperglycemia was present in $19.3 \%$ $(23 / 119)$ of pregnant women with reproductive problems versus $13.1 \%(56 / 428)$ of women without reproductive problems. Hyperglycemia up to 24 g.w. was present in $7.6 \%$ $(9 / 119)$ in pregnant women with reproductive problems against $4.7 \%$ (20/428) in those without reproductive problems, and Hyperglycemia after 24 g.w. resp. in $11.8 \%(14 / 119)$ against $8.4 \% 36 / 428)$. In all three cases, the differences are not significant.

\subsection{Adverse Outcome in Previous Pregnancy}

An adverse outcome of a previous pregnancy was found in $23.7 \%(130 / 547)$ of the examined pregnant women. Hyperglycemia was present in $17.7 \%$ (23/130) of pregnant women with an unfavorable outcome from a previous pregnancy against $13.4 \%$ (56/417) without an unfavorable outcome. Hyperglycemia up to 24 g.w. was present in $6.2 \%$ $(8 / 130)$ of pregnant women with a previous unfavorable outcome of their pregnancy against 5.0\% (21/417) in pregnant women without similar problems. The incidence of Hyperglycemia after 24 g.w. in the two compared groups with and without unfavorable outcome of previous pregnancy was $11.5 \%(15 / 130)$ resp. $8.4 \%(35 / 417)$. In all three cases the differences are not significant.

\section{Discussion}

All international guidelines focus in detail on the risk factors for the development of GDM [1, 2, 4]. There are several main factors, such as advanced maternal age, weight, preceding GDM and family history. In addition to these risk factors, there are others which also play a significant role in the development of glucose intolerance and GDM. They are subject of the present analyses.

It is noteworthy that the incidence of Hyperglycemia increases with each subsequent pregnancy and only the small number of cases in each subgroup is the reason why the importance of this factor is difficult to prove (from $14.3 \%$ in the first to $36.8 \%$ in the fourth pregnancy, NS). It should be noted that each subsequent pregnancy occurs at an older age of the woman when her weight usually increases. Therefore, in the fourth consecutive pregnancy, women are already older 
and heavier than those in the first pregnancy. These two main factors - age and obesity - are well known reasons for "diabetes epidemic" in the world [1], and we have proven their importance in our population survey from 2012 [17]. In recent months, in our previous study on the same group of 547 Bulgarian pregnant women, we proved a significant relationship between Hyperglycemia, weight and age. It was in the group with Hyperglycemia that pregnant women had a higher BMI (Mean Rank 262.48 vs. 342.26 - Mann-Whitney Test, $\mathrm{P}<0.001$ ), and were older (Mean Rank 320.84 vs 266.09 - Mann-Whitney Test, $\mathrm{P}<0.005)$ compared with the corresponding groups with Normoglycemia [18]

The close link between arterial hypertension (AH) and diabetes is well-known. In $2.4 \%(13 / 547)$ of the studied pregnant women in this project, $\mathrm{AH}$ was found during pregnancy. Hyperglycemia was found in $38.5 \%(5 / 13)$ pregnant women with $\mathrm{AH}$ versus $13.9 \%$ (74/534) in pregnant women with normal blood pressure, $\mathrm{P}<0.028$. For us, this relationship is not surprising, given our population studies from 2012 of 2032 people (956 men, 1076 women), with a mean age $49.30 \pm 14.75$ years (20-80 years). We found, after applying Multinomian Logistic Regression analysis for assessment of the relationship between the individual risk factor and the clinical manifestation of diabetes mellitus, that among Bulgarian population $\mathrm{AH}$ has a significant impact OR-4.585, 95\% CI: 3.002-7.003, P $<0.001$. Compared with the other risk factors for diabetes (obesity, TGL, HDL-c) it turned out that the most powerful risk factor for development of diabetes among Bulgarian population is AH [19].

The birth of a macrosomic baby weighing $4.5 \mathrm{~kg}$ or above in a previous pregnancy is indicated in all International guidelines as one of the main risk factors for the development of glucose intolerance and Hyperglycemia [1, 2, 4].

Low or high fetal birth weights are considered to be risk factors for GDM because they are associated with insulin resistance (IR). In general, pregnancy is a condition in which there is high metabolic activity and maintenance of glucose homeostasis is of paramount importance [14]. Increased placental transport of glucose, amino acids and fatty acids stimulates the fetus to produce more insulin and insulin-like growth factor 1 (IGF-1). This leads to its overgrowth and macrosomia at birth [20]. In most cases, $\beta$-cells in the mother's pancreas fail to compensate for the chronic excess of fuel, which leads to an increase in IR, hyperglycemia and increased glucose flow to the growing fetus. The level of physiological IR during pregnancy increases significantly and studies have shown a decrease in glucose uptake by $54 \%$ in pregnant women with GDM compared to those with normal pregnancy [21]. A macrosomic baby weighing $4.5 \mathrm{~kg}$ or above in a previous pregnancy means that there have been similar metabolic disorders, and apparently this woman has glucose intolerance, or that the present unstable compensation could be quickly and easily disrupted by the next pregnancy.

In our material, this phenomenon is rarely observed in Bulgarian pregnant women - only 3.29\% (18/547). In women who gave birth to a macrosomic baby weighing $4.5 \mathrm{~kg}$ or above in $38.9 \%(7 / 18)$ there was Hyperglycemia against $13.6 \%$
$(72 / 529)$ in women who gave birth to a fetus under $4 \mathrm{~kg}$ in a previous pregnancy, $\mathrm{P}<0.008$. It should also be noted that previous macrosomia is significant up to 24 g.w. $-22.2 \%(4 / 18)$ against $4.7 \%$ (25/529), $\mathrm{P}<0.012$, while after 24 g.w. it has no clinical significance $-16.7 \%$ (3/18) against $8.6 \%$ (47/547), NS. Therefore, we will conclude that the birth of a large fetus is a significant risk factor for the development of Hyperglycemia mainly up to 24 g.w. in the next pregnancy. It may also be risky for the development of disorders in glucose tolerance during the next pregnancy.

It is known that IR develops physiologically during pregnancy. Androgens generally play a very significant adverse role in the realization of insulin action in tissues, which impairs glucose homeostasis. Four separate phenotypes of Polycystic Ovary Syndrome (PCOS) have been identified, according to the Rotterdam criteria [22], as in $3 / 4$ of them there is hyperandrogenism. It is on this basis that each phenotype has a different severity of risk in terms of metabolic and reproductive effects. It turned out that almost $60 \%$ of our population has phenotype which includes hyperandrogenism, chronic anovulation, ultrasound data for polycystic ovaries [23]. It is hyperandrogenism that causes more severe IR. It is believed that IR and subsequent compensatory hyperinsulinemia may directly induce the flow of cellular androgen production. In turn, under the conditions of hyperandrogenization, the insulin receptor is suppressed and the expression of glucose transporters is reduced, which increases IR, ie. a vicious circle is formed. PCOS is common and affects about $12-21 \%$ of women at reproductive age [24]. In the studied 547 pregnant women data for PCOS were obtained in $11.2 \%(61 / 547)$ of them. It was found that $11.4 \%$ (9/79) of the pregnant women with PCOS had Hyperglycemia, while $11.1 \% \quad(52 / 468)$ of the rest with PCOS had Normoglycemia, NS. Therefore, the frequency is identical, but we have only anamnestic data for PCOS, and no data on the current androgen level in the studied 547 pregnant women.

Regarding hirsutism, it was registered in 7.9\% (43/547) of the studied pregnant women and it turned out that it was twice as common in pregnant women with Hyperglycemia - 13.9\% $(11 / 79)$ in comparison with pregnant women with Normoglycemia $-6.8 \%(32 / 468), \mathrm{P}<0.049$. As already noted, androgens play a very significant adverse role in the realization of insulin action in tissues. This effect is added to the physiological development of IR during pregnancy, which further impairs glucose homeostasis. This develops glucose intolerance and leads to Hyperglycemia in the pregnant women complicated by hirsutism.

Applying the nonparametric correlation coefficients of C. E. Spearman found that in the studied pregnant women there was a significant correlation between Hyperglycemia and gestational age $(\mathrm{P}<0.006)$, with a previous birth of a fetus over $4 \mathrm{~kg}(\mathrm{P}<0.03)$, with $\mathrm{AH}$ during the current pregnancy ( $\mathrm{P}$ $<0.01)$, with the presence of hirsutism $(\mathrm{P}<0.03)$. Hyperglycemia up to 24 g.w. there was a significant correlation with previous fetal birth over $4 \mathrm{~kg}(\mathrm{P}<0.001)$ and with the presence of PCOS $(\mathrm{P}<0.02)$, and Hyperglycemia after 24 g.w. there was a significant correlation only with $\mathrm{AH}$ 
during the current pregnancy $(\mathrm{P}<0.006)$.

\section{Conclusion}

It may be concluded that good knowledge of all risk factors associated with the development of glucose intolerance and GDM has an important role in the early diagnosis of this common disorder during pregnancy. In addition to the main factors, such as advanced maternal age, obesity, family history with diabetes, there are other factors which can also be important - AH during pregnancy, birth of a large fetus in a previous pregnancy and hirsutism. The proven significant correlation between Hyperglycemia and the listed additional factors gives them serious weight and requires that they should be included in the initial screening of pregnant women so that a correct decision to conduct additional studies in order to prove possible already impaired glucose tolerance could be taken on time.

\section{References}

[1] International Diabetes Federation. (2019). IDF Diabetes Atlas, $9^{\text {th }}$ edn. Brussels, Belgium: International Diabetes Federation.

[2] Moshe Hod, Kapur A, Sacks D. A, Hadar E, Agarwa M, Di Renzo G. C, Roura L. C, McIntyre H. D, Morris J. L, Divakar $\mathrm{H}$. The International Federation of Gynecology and Obstetrics FIGO. (2015). Initiative on gestational diabetes mellitus: A pragmatic guide for diagnosis, management, and care. International Journal of Gynecology and Obstetrics 131, S3, S173-S211.

[3] World Health Organization. (2019). Classification of Diabetes mellitus 2019. ISBN 978-92-4-151570-2.

[4] NICE. (2015). Diabetes in pregnancy: management from preconception to the postnatal period. https://www.nice.org.uk/terms-and conditions\#notice-ofrights.

[5] American Diabetes Association. (2019). Classification and diagnosis of diabetes. Diabetes Care 42 (Suppl 1): S13-S28.

[6] Yu Y, Arah O. A, Liew Z, Cnattingius S, Olsen J, Sørensen H. T, Qin G, Li J. (2019). Maternal diabetes during pregnancy and early onset of cardiovascular disease in offspring: population based cohort study with 40 years of follow-up. BMJ 367: 16398.

[7] Guerrero-Romero F, Aradillas-García C, Simental-Mendia LE, Monreal-Escalante E, de la Cruz Mendoza E, Rodríguez-Moran M. (2010). Birth weight, family history of diabetes, and metabolic syndrome in children and adolescents. J Pediatr 156 (5): 719-723.

[8] Scholtens D. M, Kuang A, Lowe L. P, Hamilton J, Lawrence J. M, Lebenthal Y, Brickman W. J, Clayton P, Ma R. C, McCance D, Tam W. H, Catalano P. M, Linder B, Dyer A. R, Lowe Jr W. L, Metzger B. E, HAPO Follow-up Study Cooperative Research Group. (2019). Hyperglycemia and adverse pregnancy outcome follow-up study (HAPO FUS): maternal glycemia and childhood glucose metabolism. Diabetes Care 42 (3): 381-392.

[9] Egerup P, Mikkelsen A. P, Kolte A. M, Westergaard D, Rasmussen S, Knop F. K, Lidegaard O, Nielsen H. S. (2020). Pregnancy loss is associated with type 2 diabetes: a nationwide case-control study. Diabetologia 63 (8): 1521-1529. doi: 10.1007/s00125-020-05154-z

[10] Sweeting AN, Ross GP, Hyett J, Molyneaux L, Constantino M, Harding A. J, Wong J. (2016). Gestational diabetes mellitus in early pregnancy: evidence for poor pregnancy outcomes despite treatment. Diabetes Care 39 (1): 75-81. https://doi.org/10. 2337/dc15-0433.

[11] Elliott H. R, Sharp G. C, Relton C. L, Lawlor D. A. (2019). Epigenetics and gestational diabetes: a review of epigenetic epidemiology studies and their use to explore epigenetic mediation and improve prediction. Diabetologia 62: 21712178.

[12] McCance D, Maresh M, Sacks DA. A practical manual of diabetes in pregnancy. John Wiley \& Sons, 2017. doi: $10.1002 / 9781119043805$.

[13] Ferrara A. (2007). Increasing prevalence of gestational diabetes mellitus: a public health perspective. Diabetes Care 30 (Suppl 2): S141-146. doi: 10.2337/dc07-s206.

[14] Plows J. F, Stanley J. L, Baker P. N, Reynolds C. M, Vickers M. H. (2018). The Pathophysiology of Gestational Diabetes Mellitus. Int. J. Mol. Sci. 19, 3342. doi: 10.3390/ijms19113342.

[15] World Medical Association (2013). "Declaration of Helsinki: Ethical Principles for Medical Research Involving Human Subjects". JAMA. 310 (20): 2191-2194. doi: 10.1001/jama.2013.28105.

[16] Williams B, Mancia G, Spiering W, Rosei E. A, Azizi M, Burnier M, Clement D. L, Coca A, de Simone G, Dominiczak A, Kahan T, Mahfoud F, Redon J, Ruilope L, Zanchetti A, Kerins M, Kjeldsen S. E, Kreutz R, Laurent S, Lip G. Y. H, McManus R, Narkiewicz K, Ruschitzka F, Schmieder R. E, Shlyakhto E, Tsioufis C, Aboyans V, Desormais I. (2018). ESC/ESH Guidelines for the management of arterial hypertension. The Task Force for the management of arterial hypertension of the European Society of Cardiology (ESC) and the European Society of Hypertension (ESH).

[17] Borissova A-M, Shinkov A, Kovatcheva R, Vlahov J, Dakovska L, Todorov T. (2015). Changes in the Prevalence of Diabetes Mellitus in Bulgaria, 2006-2012. Clinical Medicine Insights: Endocrinology and Diabetes 8, 41-45.

[18] Borissova A-M, Trifonova B, Dakovska L, Michaylova E, Vukov M. (2021). Age, obesity, family history, previous Gestational diabetes are major risk factors for Hyperglycemia in pregnant Bulgarian women. European Journal of Preventive Medicineл 9 (2): 39-45.

[19] Borissova A-M, Shinkov A, Vlahov J, Dakovska L, Svinarov D, Kovatcheva R, Krivoshiev S, Popov A, Mihaylov G, Koteva A, Vukov M, Todorov T, Kalinov K, Kasabova L, Atanassova I, Aslanova N. (2016). Prevalence and role of risk factors on the onset of diabetes and prediabetes in Bulgarian population. Epidemiology of Endocrine disorders in Bulgaria, 2006-2012, 201-220 In: Epidemiology of Endocrine disorders in Bulgaria 2006-2012. Editor Prof. Anna-Maria Borissova. PARADIGMA Ltd. 2016 ISBN 978-954-326-291-5.

[20] Schwartz R, Gruppuso P. A, Petzold K, Brambilla D, Hiilesmaa $\mathrm{V}$, Teramo K. A. (1994). Hyperinsulinemia and macrosomia in the fetus of the diabetic mother. Diabetes Care. 17, 640-648.

[21] Catalano, P. M. (2014). Trying to understand gestational diabetes. Diabet. Med. 31, 273-281. 
[22] Kristensen S. L, Ramlau-Hansen CH, Ernst E, Olsen S. F, Bonde J. P, Vested A, Toft G. (2010). A very large proportion of young Danish women have polycystic ovaries: is a revision of the Rotterdam criteria needed? Hum Reprod 25: 3117-3122. doi: 10.1093/humrep/deq273.

[23] Orbetzova M. (2007). Polycystic ovary syndrome (PCOS).
Diagnosis, differential diagnosis, treatment. Science Endocrinology. 2, 57-60.

[24] Boyle J, Teede H. J. (2012). Polycystic ovary syndrome. Australian Family Physician. 41 (10): 752-756. 